Published in final edited form as:

Leuk Lymphoma. 2020 June ; 61(6): 1508-1511. doi:10.1080/10428194.2020.1725505.

\title{
A retrospective study on prephase therapy prior to definitive multiagent chemotherapy in aggressive lymphomas
}

\author{
Luis Malpica ${ }^{\mathrm{a}}$, Bolanle Mufuka ${ }^{\mathrm{b}}$, Jonathan Galeotti ${ }^{\mathrm{c}}$, Xianming Tan ${ }^{\mathrm{d}}$, Natalie Grover ${ }^{\mathrm{a}}$, \\ Stephen M. Clark ${ }^{\mathrm{e}}$, Anne Beaven ${ }^{\mathrm{a}}$, Hyman Muss ${ }^{\mathrm{a}}$, Christopher Dittus ${ }^{\mathrm{a}}$ \\ aDivision of Hematology-Oncology, Department of Medicine, University of North Carolina, Chapel \\ Hill, NC, USA; \\ bepartment of Internal Medicine, University of North Carolina, Chapel Hill, NC, USA; \\ 'Department of Pathology, University of North Carolina, Chapel Hill, NC, USA; \\ dDepartment of Biostatistics Shared Resource, Lineberger Comprehensive Cancer Center, \\ University of North Carolina, Chapel Hill, NC, USA; \\ eDepartment of Pharmacy, University of North Carolina, Chapel Hill, NC, USA
}

\begin{abstract}
Diffuse large B-cell lymphoma (DLBCL) and Burkitt lymphoma (BL) are aggressive nonHodgkin's lymphomas (NHLs) that evolve rapidly and are fatal if untreated [1]. While the majority of patients are successfully initiated on multiagent chemotherapy, there is a subgroup of patients, particularly elderly patients and those with high disease burden, who are at risk for developing treatmentrelated toxicities during the initial phase of therapy [2]. Prephase therapy, a regimen consisting of corticosteroids with or without low-dose chemotherapy, has been used as a preventive strategy prior to definitive multiagent chemotherapy to ameliorate the development of toxicity, such as febrile neutropenia, tumor lysis syndrome (TLS), deterioration in performance status (PS), and death; however, data regarding the efficacy of this approach is limited [2-4]. We present data from our experience at the University of North Carolina Cancer Hospital, NC, U.S.A. on the safety and efficacy of prephase therapy prior to definitive multiagent chemotherapy in patients with aggressive NHLs.
\end{abstract}

We conducted a retrospective analysis of patients 18 years and older with newly diagnosed DLBCL or BL between January 2014 and December 2017. Patients were identified via the Carolina Data Warehouse for Health database per Institutional Review Board-approved protocol. Patients with primary central nervous system lymphoma, or previously diagnosed with an indolent or low-grade lymphoma that transformed to an aggressive NHL were

\footnotetext{
CONTACT Christopher Dittus, chris_dittus@med.unc.edu, Division of Hematology and Oncology, Department of Medicine, University of North Carolina, 170 Manning Drive, CB\# 7305, Chapel Hill, NC, 27599, USA.

Author contributions

L.M., and C.D., designed the research; L.M., B.M., J.G., X.T., and C.D., extracted the data, and made the table; L.M., B.M., J.G.,

N.G., X.T., S.C., A.B., H.M., and C.D., analyzed results and/or wrote the paper.

Disclosure statement

N.G. and C.D., disclose Seattle Genetics consultancy.

L.M., B.M., J.G., X.T., S.C., A.B., and H.M., declare no competing financial interests.
} 
excluded. Pre-therapy clinical data such as patient's performance status (ECOG PS score), absolute neutrophil count (ANC), platelet count, lactate dehydrogenase (LDH) level, disease stage, International Prognostic Index (IPI) score, bulky disease (maximal transverse diameter of $>10 \mathrm{~cm}$ ), and bone marrow involvement (evaluated by either bone marrow biopsy or positron emission tomography) were obtained through chart review.

Patients were divided into 'prephase' (PP) and 'nonprephase' (NPP) groups with both groups evaluated until day 30. The ANC nadir, platelet nadir, and grade 3-4 cytopenias (defined as hemoglobin $<8 \mathrm{~g} / \mathrm{dL}$, ANC $<1 \times 10^{3}$ cells $/ \mu \mathrm{L}$, and platelets $<50 \times 10^{3}$ cells $/ \mu \mathrm{L}$ ) were recorded post cycle 1 of multiagent chemotherapy. Of those who developed grade 3-4 cytopenias, the time to recovery from grade 3-4 cytopenias was determined from the time of first cytopenia occurrence to a hemoglobin $\geq 8 \mathrm{~g} / \mathrm{dL}$, ANC $\geq 1 \times 10^{3}$ cells $/ \mu \mathrm{L}$, and platelet count $250 \times 10^{3}$ cells $/ \mu \mathrm{L}$. Episodes of febrile neutropenia, tumor lysis syndrome, hospital admission (or readmission if patient received initial therapy as inpatient), emergency visits, and 30-day mortality were obtained. Clinical characteristics, definitive chemotherapy regimens and patient outcomes were reported using descriptive statistics for all subjects and stratified by therapy group (PP versus non-PP group). We compared these variables between $\mathrm{PP}$ group and non-PP group using the Wilcoxon rank sum test (for numerical variables) or Chi-square test (for categorical variables). Wilcoxon signed rank test was used to test whether there was significant change in PS from prePP to post-PP. All tests were two-sided at significance level of 0.05 , unless otherwise specified. Statistical analyses were performed using SAS 9.4 (SAS Institute, Cary, NC) software.

A total of 92 patients included in the analyses had a diagnosis of DLBCL $(n=82,89 \%)$ or BL $(n=10,11 \%)$. Twenty-two patients (24\%) received PP therapy, and $70(76 \%)$ did not. The median age at diagnosis was 61 years with a female predominance in both groups (see Table 1 for clinical characteristics). Baseline poor ECOG PS and higher IPI score were more common in the PP group compared to the NPP group (median ECOG PS score 2 vs. 0, and median IPI score 3 vs. 2 , respectively; both $p<.01$ ). A baseline LDH level of more than 1.5 times the upper limit of normal (ULN) was more common in the PP group compared to the NPP group ( $n=15,68 \%$; versus $n=17,19 \%$, respectively, $p<.01$ ). Stage III-IV disease, bulky disease, and bone marrow involvement were not statistically significant between the two groups, nor were the median ANC and platelet count at diagnosis (see Table 1).

Single-agent oral prednisone, either at $1 \mathrm{mg} / \mathrm{kg}$ oral daily for 5 days $(n=11)$ or flat dose of $100 \mathrm{mg}$ oral daily for 5 days $(n=6)$, was the most commonly used prephase regimen. Five patients received combination therapy of prednisone at $100 \mathrm{mg}$ oral daily from day -6 to day 0 , and either: vincristine $1 \mathrm{mg}$ intravenously on day $-6(n=1)$, cyclophosphamide 200 $\mathrm{mg} / \mathrm{m}^{2}$ on day $-6(n=1)$, or vincristine plus cyclophosphamide $(n=3)$. All regimens were well tolerated. The PP group had a significant improvement in performance status from a median performance status of 2 at baseline to a median performance status of $1(p<.01)$ by day 4 of definitive multiagent chemotherapy. The NPP group did not have a significant change in performance status (Table 1). There was no difference in the degree of grade 3-4 cytopenias. Both groups had the same median ANC nadir $\left(0.6 \times 10^{3}\right.$ cell $\left./ \mathrm{mm}^{3}\right)$, however, the PP group had a lower platelet nadir compared to the NPP group $\left(80\right.$ vs. $150 \times 10^{3}$ cells $/ \mu \mathrm{L}$, respectively, $p=.03$ ). The PP group had a shorter time for ANC and platelet count recovery 
compared to the NPP group (ANC 4 vs. 8 days, $p=.01$; platelet 4 vs. 9 days, $p=.02$ ). Additionally, the PP group had a significant reduction in the number of patients with elevated LDH post definitive multiagent chemotherapy (baseline LDH $>1.5 \times \mathrm{ULN}, n=15$, $68 \%$ versus $n=5,23 \%$ at day 4 post-chemotherapy; $p<.01)$ compared to the NPP group ( $n$ $=17,19 \%$ versus $n=13,14 \%$, respectively; $p=.81$ ). There was no significant difference in the episodes of febrile neutropenia, tumor lysis syndrome, hospitalization, emergency visits, or 30-day mortality. Only one patient, in the NPP group, died during the evaluation period (a 70-year-old man with DLBCL who developed bacteremia at day 29 post-first cycle).

Our analysis demonstrates that in aggressive lymphomas, prephase therapy significantly improved performance status by day 4 of definitive multiagent chemotherapy at first cycle, even in patients with a high IPI score and elevated LDH. Additionally, our study focused predominantly on a chemotherapy-free prephase regimen, with 17 of 22 (77\%) patients receiving prednisone alone. While our findings are in-line with previously reported results, these studies have focused on prephase regimens that include chemotherapy [2-4].

Prephase therapy was initially implemented into the chemotherapy protocols of pediatric patients with acute lymphoblastic leukemia (ALL) [5]. Subsequently, clinicians extrapolated this approach from ALL to the management of NHL. The NHL-B2 trial, was the first to utilize prephase therapy in order to prevent the 'first-cycle effect' (described as deepest ANC nadir, longest duration of neutropenia, and highest rate of therapy-associated deaths) [6]. Prephase therapy originally consisted of a single dose of vincristine at $1 \mathrm{mg}$ plus 7 days of oral prednisone before the first cycle of CHOP. [2] The same researchers report that, more recently, vincristine was removed without a change in the positive prephase treatment effect; unfortunately, though, they do not provide specific, prednisone-only, data to support this conclusion. Despite this, an improvement of the performance status after several days of prephase was described, and this approach became popular among oncologists in certain regions, including Germany. In fact, many oncologists will give a course of prephase therapy to all DLBCL patients, adapting the duration of prephase to the patient's performance status [2]. Although successive trials such as MabThera International Trial (MInT) [7], RICOVER-60 [8], SMARTE-R-CHOP-14 [9], and LNH09-7B [10], included prephase therapy within their protocols, specific data regarding the frequency and duration of cytopenias, tumor lysis syndrome development, hospital admissions, among other clinical factors were not reported.

While a recent prospective study on prephase therapy showed an improvement in performance status and reduction in the number of febrile neutropenia cases compared to the non-prephase cohort, this study used a chemotherapy-based prephase approach (prednisone plus vincristine) [3]. Additionally, this study did not include aggressive histologies outside of DLBCL. Our study demonstrates that a chemotherapy-free approach (i.e. single-agent prednisone) improves performance status, reduces LDH, and shortens the ANC and platelet nadir. We also included patients with Burkitt lymphoma, showing that these positive effects are not only seen in patients with DLBCL. While we did not show a significant reduction in febrile neutropenia, we do describe a significant reduction in the length of grade 3-4 neutropenia, which has been shown to be associated with the risk of febrile neutropenia and infections in patients with nonmyeloid malignancies [11]. 
In conclusion, our study reports that the administration of a chemotherapy-free prephase regimen (singleagent prednisone) in patients with DLBCL and Burkitt lymphoma is associated with improved performance status, reduction in $\mathrm{LDH}$, and a shorter length of neutropenia and thrombocytopenia with the first cycle of chemotherapy. We recommend consideration of a prednisone prephase regimen prior to chemotherapy for patients with a poor performance status at baseline and those at risk for a 'first treatment effect'.

\section{References}

[1]. Rovira J, Valera A, Colomo L, et al. Prognosis of patients with diffuse large B cell lymphoma not reaching complete response or relapsing after frontline chemotherapy or immunochemotherapy. Ann Hematol. 2015;94(5):803-812. [PubMed: 25501975]

[2]. Pfreundschuh M How I treat elderly patients with diffuse large B-cell lymphoma. Blood. 2010;116(24):5103-5110. [PubMed: 20805363]

[3]. Lakshmaiah KC, Asati V, Babu KG, et al. Role of prephase treatment prior to definitive chemotherapy in patients with diffuse large B-cell lymphoma. Eur J Haematol. 2018;100(6): 644-648. [PubMed: 29569279]

[4]. Cui Y, Li X, Sun Z, et al. Safety and efficacy of low-dose prephase before conventional-dose chemotherapy for ulcerative gastric diffuse large B-cell lymphoma. Leuk Lymphoma. 2015; 56(9):2613-2618. [PubMed: 25676238]

[5]. Hyman CS. Prednisone therapy of acute lymphatic leukemia in children. Cancer. 1956;9(5):965970. [PubMed: 13364883]

[6]. Pfreundschuh M, Trümper L, Kloess M, et al. Two-weekly or 3-weekly CHOP chemotherapy with or without etoposide for the treatment of elderly patients with aggressive lymphomas: results of the NHL-B2 trial of the DSHNHL. Blood. 2004;104(3):634-641. [PubMed: 15016643]

[7]. Pfreundschuh M, Trümper L, Österborg A, et al. CHOP-like chemotherapy plus rituximab versus CHOP-like chemotherapy alone in young patients with good-prognosis diffuse large-B-cell lymphoma: a randomised controlled trial by the MabThera International Trial (MInT) Group. Lancet Oncol. 2006;7(5):379-391. [PubMed: 16648042]

[8]. Pfreundschuh M, Schubert J, Ziepert M, et al. Six versus eight cycles of bi-weekly CHOP-14 with or without rituximab in elderly patients with aggressive CD20+ B-cell lymphomas: a randomised controlled trial (RICOVER-60). Lancet Oncol. 2008;9(2):105-116. [PubMed: 18226581]

[9]. Pfreundschuh M, Poeschel V, Zeynalova S, et al. Optimization of rituximab for the treatment of diffuse large b-cell lymphoma (II): extended rituximab exposure time in the SMARTERCHOP-14 trial of the German high-grade non-Hodgkin lymphoma study group. J Clin Oncol. 2014;32(36):4127-4133. [PubMed: 25403207]

[10]. Peyrade F, Serge B, Delwail V, et al. Pre-phase chemotherapy followed by ofatumumab (OFA) and reduced dose CHOP (OFA-mini-CHOP) for patients over 80 years with diffuse large B-cell lymphoma (DLBCL) - a lymphoma study association (LYSA) prospective phase II study (LNH09-7B). Blood. 2014; 124(21):3042-3042.

[11]. Li Y, Klippel Z, Shih X, et al. Relationship between severity and duration of chemotherapyinduced neutropenia and risk of infection among patients with nonmyeloid malignancies. Support Care Cancer. 2016;24(10):4377-4383. [PubMed: 27278272] 
Table 1.

Clinical features, chemotherapy regimen, and outcomes of aggressive non-Hodgkin lymphoma patients.

\begin{tabular}{|c|c|c|c|c|}
\hline Category & Prephase $(n=22)$ & Non-prephase $(n=70)$ & Total $(n=92)$ & $p$ value \\
\hline Age, median in years (range) & $59(21-87)$ & $61(20-85)$ & $61(20-87)$ & .96 \\
\hline Gender, $n(\%)$ & & & & .34 \\
\hline Female & $13(59)$ & $38(54)$ & $51(55)$ & - \\
\hline Male & $7(41)$ & $32(46)$ & $39(45)$ & - \\
\hline Diagnosis, $n(\%)$ & & & & .08 \\
\hline Burkitt & $4(18)$ & $6(9)$ & $10(11)$ & - \\
\hline DLBCL & $18(82)$ & $64(91)$ & $82(89)$ & - \\
\hline ANC at diagnosis (median) & $5.25 \times 10^{3}$ cells $/ \mu \mathrm{L}$ & $4.9 \times 10^{3}$ cells $/ \mu \mathrm{L}$ & $5 \times 10^{3}$ cells $/ \mu \mathrm{L}$ & .19 \\
\hline Platelet count at diagnosis (median) & $250 \times 10^{3}$ cells $/ \mu \mathrm{L}$ & $289 \times 10^{3}$ cells $/ \mu \mathrm{L}$ & $270 \times 10^{3} \mathrm{cells} / \mu \mathrm{L}$ & .17 \\
\hline $\mathrm{LDH}(1.5 \times \mathrm{ULN})$ at diagnosis, $n(\%)$ & $15(68)$ & $17(19)$ & $32(35)$ & $<.01$ \\
\hline Stage, $n(\%)$ & & & & .41 \\
\hline I-II & $7(32)$ & $34(49)$ & $41(45)$ & - \\
\hline III-IV & $15(68)$ & $36(51)$ & $51(56)$ & - \\
\hline IPI score (median) & 3 & 2 & 2 & $<.01$ \\
\hline Bulky disease, $n(\%)$ & $7(32)$ & $11(16)$ & $18(20)$ & .11 \\
\hline Bone marrow involvement, $n(\%)^{a}$ & $4(18)$ & $5(7)$ & $9(10)$ & .09 \\
\hline \multicolumn{5}{|l|}{ Prephase regimen, $n(\%)$} \\
\hline Prednisone & $17(77)$ & - & - & - \\
\hline VP regimen & $1(5)$ & - & - & - \\
\hline $\mathrm{CP}$ regimen & $1(5)$ & - & - & - \\
\hline CVP regimen & $3(13)$ & - & - & - \\
\hline Chemotherapy regimen, $n(\%)$ & & & & .07 \\
\hline $\mathrm{R}-\mathrm{CHOP}$ & $9(41)$ & $47(67)$ & $56(61)$ & - \\
\hline R-miniCHOP & $2(8)$ & $3(4)$ & $5(5)$ & - \\
\hline R-EPOCH & $9(41)$ & $19(28)$ & $28(31)$ & - \\
\hline R-HyperCVAD & $1(5)$ & $1(1)$ & $2(2)$ & - \\
\hline R-CEOP & $1(5)$ & 0 & $1(1)$ & - \\
\hline GSCF use during cycle 1 & $9(41)$ & $32(46)$ & $41(45)$ & .82 \\
\hline \multicolumn{5}{|l|}{ ECOG PS, median (range) } \\
\hline At baseline & $2(1-3)$ & $0(0-2)$ & $1(0-3)$ & $<.01$ \\
\hline At day 4 post-first cycle, $p$ value & $1(0-1), p<.01$ & $1(1-3)$ & $1(0-3)$ & .81 \\
\hline \multicolumn{5}{|l|}{ Grade $3-4$ cytopenia, $n(\%)^{b}$} \\
\hline Neutropenia & $12(54)$ & $26(37)$ & 38 & .13 \\
\hline Thrombocytopenia & $6(27)$ & $10(14)$ & 16 & .12 \\
\hline Anemia & $5(22)$ & $9(13)$ & 14 & .19 \\
\hline ANC nadir (median) & $0.6 \times 10^{3}$ cells $/ \mu \mathrm{L}$ & $0.6 \times 10^{3}$ cells $/ \mu \mathrm{L}$ & $0.6 \times 10^{3}$ cells $/ \mu \mathrm{L}$ & .95 \\
\hline Platelet nadir (median) & $80 \times 10^{3}$ cells $/ \mu \mathrm{L}$ & $150 \times 10^{3}$ cells $/ \mu \mathrm{L}$ & $120 \times 10^{3}$ cells $/ \mu \mathrm{L}$ & .03 \\
\hline LDH $(1.5 \times$ ULN $)$ by day 4 post-first cycle, $n(\%)$ & $5(23)$ & $13(14)$ & $18(20)$ & .07 \\
\hline
\end{tabular}




\begin{tabular}{lcccc}
\hline Category & Prephase $(\boldsymbol{n}=\mathbf{2 2})$ & Non-prephase $(\boldsymbol{n}=\mathbf{7 0})$ & Total $(\boldsymbol{n}=\mathbf{9 2})$ & $\boldsymbol{p}$ value \\
\hline Number of days until count recovery (median) & & & & \\
$\quad$ ANC & 4 & 8 & 4 & .01 \\
$\quad$ Platelet & 4 & 9 & 4 & .02 \\
Febrile neutropenia, $n(\%)$ & $1(5)$ & $1(1)$ & $2(2)$ & .74 \\
Tumor lysis syndrome & $3(14)$ & $2(3)$ & $5(5)$ & .07 \\
Admission within 30 days, $n(\%)$ & & & & .16 \\
$\quad$ Hospitalization & $1(5)$ & $12(17)$ & $13(14)$ & .17 \\
Emergency room visit & $1(5)$ & $11(16)$ & $12(13)$ & .59 \\
30-Day mortality, $n(\%)$ & 0 & $1(1)$ & $1(1)$ &
\end{tabular}

ANC: Absolute neutrophil count; CP: Cyclophosphamide and prednisone; CVP: Cyclophosphamide, vincristine and prednisone; DLBCL: Diffuse large B-cell lymphoma; ECOG PS: Eastern Cooperative Oncology Group Performance status; GCSF: Granulocyte colony stimulating factor; HyperCVAD: cyclophosphamide, vincristine, doxorubicin and dexamethasone, alternated with high-dose cytarabine and methotrexate; IPI: International Prognostic Index; R-CHOP: cyclophosphamide, vincristine, doxorubicin and prednisone; R-miniCHOP: reduced cyclophosphamide and doxorubicin doses; R-CEOP: Etoposide substituted for doxorubicin (H); R-EPOCH: dose-adjusted etoposide cyclophosphamide, vincristine, doxorubicin and prednisone; VP: Vincristine and prednisone.

${ }^{a}$ Bone marrow involvement was evaluated by either bone marrow biopsy or positron emission tomography.

${ }^{b}$ Grade 3-4 cytopenias were defined as hemoglobin $<8 \mathrm{~g} / \mathrm{dL}$, ANC $<1 \times 10^{3}$ cells $/ \mu \mathrm{L}$, and platelets $<50 \times 10^{3}$ cells $/ \mathrm{mL}$, and assessed anytime post cycle 1 of multiagent chemotherapy. 